\title{
Modeling seizures: from single neurons to networks
}

Damien Depannemaecker ${ }^{1, \#}$, Alain Destexhe ${ }^{1}$, Viktor Jirsa ${ }^{2}$, Christophe Bernard ${ }^{2}$

${ }^{1}$ Paris-Saclay University, French National Centre for Scientific Research (CNRS), Institute of Neuroscience (NeuroPSI), 91198 Gif sur Yvette, France

${ }^{2}$ Aix Marseille Univ, INSERM, INS, Institut des Neurosciences des Systèmes, Marseille, France

\#Corresponding author: damien.d@cnrs.fr

\section{Abstract}

Dynamical system tools offer a complementary approach to detailed biophysical seizure modeling, with a high potential for clinical applications. This review describes the theoretical framework that provides a basis for theorizing certain properties of seizures and for their classification according to their dynamical properties at onset and offset. We describe various modeling approaches spanning different scales, from single neurons to large-scale networks. This narrative review provides an accessible overview of this field, including non-exhaustive examples of key recent works. Introduction

Epilepsies are defined by the occurrence of spontaneous and recurrent seizures [1]. Seizures are characterized by electrophysiological (field potential) signatures, varying from one patient to the next and even within a given patient [2]. Given such variability, it is important to rely on a taxonomy of seizures, not only for mechanistic studies (different seizures may be associated with different underlying mechanisms, hence to different pharmacotherapy) but also for diagnostic purposes (particularly for presurgical evaluation in the case of drug-resistant epilepsies). One approach to characterize seizure types is to analyze their dynamical properties [2-4]. For this, dynamical systems theory is essential $[3,5,6]$. In this journal, Stefanescu and 
colleagues [5] show the importance and the basis of the methods used for computational modeling of seizures. Building on this work, we propose here to highlight some recent works in this field. Numerous approaches emerged: from phenomenological aspects of dynamics to detailed biophysical models, from the subcellular scale to the large scale of whole brain regions [7-12]. We discuss the interest of each of these different approaches to show how all contribute to a better understanding of the mechanisms underlying seizures.

\section{Computational modeling of seizures.}

Brain activity constantly changes from one moment to the next. A key factor (among many) determining the temporal evolution of a neuron's activity is the excitability of the neuronal membrane. Thus, the vast majority of models use this variable, from the cellular level to neural networks' behavior to the brain's global activity. At any level of analysis, the type of study that can be carried out from a dynamical system point of view depends upon the number of dimensions of the model (i.e., number of variables). A model often needs to be reduced to maintain a manageable dimensionality before moving to the next level of analysis, as shown schematically in Fig 1. 


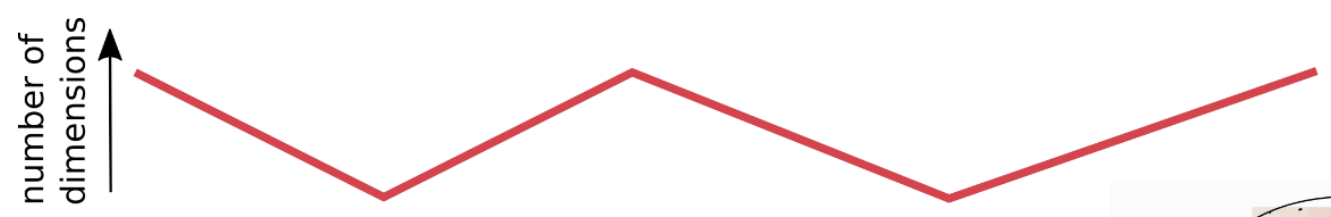

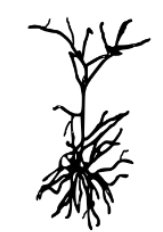

detailed neuron

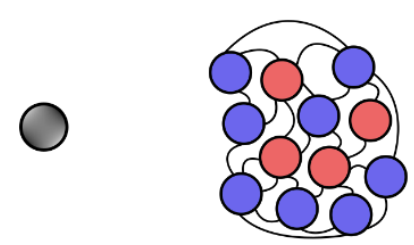

point neuron neuronal network

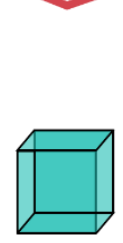

mesoscopic model

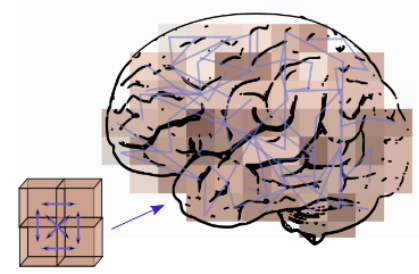

large-scale model

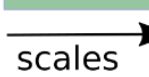

Figure 1. Model dimensions for different scales. Detailed models at a given scale have a high number of dimensions (i.e., number of variables) and can be reduced. Building a network is possible using a reduced model to get access to higher levels.

The core of a model includes sources of electrophysiological activity to produce the readout of the model; here electrographical seizures. Some models include other variables that interact with electrophysiological activity, such as metabolic activity, ion or oxygen concentration variations, or the interaction with glial cells.

Another approach consists of modeling the signal itself (i.e., the readout) that can be experimentally recorded by different methods such as electroencephalography (EEG), magnetoencephalography (MEG), and functional magnetic resonance imagery (fMRI). Since, in these cases, it is the measurement that is modeled and not the source, it becomes difficult to obtain insight in terms of biological mechanisms. The mapping between source and sensor signals is one of the most difficult question when interpreting human brain imaging signals. The Virtual Brain (TVB) simulator provides an answer to some degree $[13,14]$, even though principled model non-identifiability cannot be overcome by definition. The TVB approach has the advantage of remaining as close as possible to the clinical data. 
It is, however, possible to establish a link between the source and the signal [15], which requires keeping track of the temporal evolution of certain variables. A large majority of models used in computational neuroscience are based on systems of differential equations to capture such temporal evolutions. In a first-order differential system, the state of a variable at a time $t$ is calculated based on its variation with respect to time $\mathrm{t}-\Delta \mathrm{t}$. The rate of amplitude changes over time will determine the time scale. Each equation composing the system can vary according to its own time scale. This notion of time scales is an important consideration in epilepsy because spontaneous seizures occur on a much slower scale (hour, day, month) than that of the electrophysiological signature of a seizure itself (seconds). Thus, to model epilepsy, a so-called rapid system is needed, switching from the "normal" state to a seizure state. A slower system is needed to drive the transition between these states. It is possible to construct a slow-fast system [16,17], including these two time scales, or an external input can drive the transition between states. Such models can produce the different states, or in other words, dynamical regimes. These dynamical regimes can be observed in the phase space. The phase space is an abstract space with a number of dimensions corresponding to the number of variables. A vector given by the value of the differential of each variable exists for each point of the phase space. It gives the direction and strength in which the trajectory will be attracted if it goes through this point. A change in parameter values modifies the structure of the phase space. At a given point, if all differentials are equal to zero, then it is a fixed point. The disappearance or the birth of a fixed point due to changes in parameter values is called a bifurcation. Some preferential paths or regions can be identified in this space. These are attractors, shaping the volume in which the trajectory will evolve. A change of dynamic regime of the system (from awake to seizure, for example) can be considered 
as a change of attractor. Either an attractor evolves (one disappears, one appears), or two attractors co-exist in the phase space, and the trajectory passes from one to the other, crossing a separatrix. When the system has few dimensions, these attractors are simple objects, such as fixed points or limit-cycles. This is one of the advantages provided by the reduced dimensional phenomenological models described in the next section.

\section{Phenomenological models of seizure}

The phenomenological approach, reducing the models to a minimum number of variables and parameters, enables an exhaustive study of the dynamics. The Epileptor is one such model [3]. It was constructed to reproduce the most common electrophysiological signature of seizures found in patients and experimental models $[3,18,19]$. Interestingly, the Epileptor model also includes in its dynamical regime status epilepticus and depolarization block $[18,19]$. It is possible to generate sixteen possible seizure types in terms of dynamics or dynamotypes [2,4]. Each of these classes differs according to the type of transition between the healthy state and the seizure state. In terms of dynamical systems theory, this corresponds to the types of bifurcation at the onset and the offset of the seizure $[3,19]$. Each bifurcation can be displayed on a map of brain dynamics $[2,4]$. Thus, this theoretical approach not only leads to a taxonomy of clinically observed seizures [2] but also offers a theoretical framework to explain the coexistence of healthy and pathological regimes. It also gives insight on how it is possible to navigate the map of brain dynamics, explaining why individual patients can display several dynamotypes [18]. This type of model, called phenomenological, precisely presents the interest of describing only the observed phenomenon, independently of the associated biophysical underpinnings. Although it does not 
directly inform the underlying biological mechanisms, it provides a theoretical framework and predictions in terms of dynamics. More detailed biophysical models can refer to this framework, from the single neuron to the large-scale brain model scales. In the next subsections, we will detail some approaches for modeling electrophysiological seizures across scales from the lowest (i.e., single neuron) to the highest (i.e., whole brain).

\section{Models at the single neuron level}

Single neuron-scale models are very diverse and can include very different levels of detail. Some models are highly detailed, taking into account sub-cellular organelles such as mitochondria [9] or the detailed morphology of the dendritic and axonal tree $[20,21]$. In the latter case, it is necessary to divide the geometry of the neuron into different compartments. As a function of the model's objectives, reducing the general dynamics to a single compartment is possible. We then speak of a point neuron model (see Fig. 1).

Many approaches are possible for this type of modeling [22]. We will see how to extend this approach to models of seizures in particular. A first approach made by Cressman and colleagues [23] proposed a model at the single neuron scale. It is based on the formalism developed by Hodgkin and Huxley [24]. It can be made more complex by adding the equations describing the variations in ionic concentrations and the interaction with glial cells, which dynamically play the role of slow variables. This model has the advantage of being detailed from a biophysical point of view. It presents a large number of differential equations limiting the possibility of fully studying its dynamics, which requires using a reduced model [23]. The reduced model reproduces only a few 
bifurcation types at the onset and offset of a seizure, but not the most commonly observed in clinical and experimental data (SN/homoclinic). Such an approach has been extended [23,25], resulting in a unified framework for spike, seizure, and spreading depression [10]. In this framework, it is possible to study specific biophysical interactions: for example, the relationship between oxygenation and electrophysiological activities [11]. At this scale, a correspondence with in-vitro experiments can be done [26].

Following the same general strategy, a different reduced model has been proposed [8]. It offers a unified framework for spiking, burst, seizure, status epilepticus, and depolarization block. It describes the membrane's excitability and the interaction with the environment (bath/glial cell) through changes in ionic concentrations with only four differential equations. Compared with the previous one, this model is less detailed biophysically. But it can establish correspondences with phenomenological models as it is dimensionally reduced. In this case, the underlying dynamics correspond to what is observed in the signals recorded experimentally.

A third approach has been proposed by Chizhov and colleagues [27], which also takes into account the dynamic constraints described by phenomenological models. The model can reproduce the different dynamics of seizures in high potassium conditions. The electrophysiological activities described in this model are not only associated with variations in ionic concentrations but also with synaptic activity. Although it is at the scale of a single neuron, the model considers the effects of the network through synaptic inputs. The network models are discussed in the next subsection. 


\section{Models at the network level}

Network models include many cells that interact with each other. If the synaptic activity is often dominant in these models, some works show that all the other interactions (ephaptic, ionic diffusion, etc.) can be sufficient for the genesis and the propagation of seizures $[28,29]$. Ephaptic interactions may, for instance, synchronize action potential propagation in tightly packed fiber bundles [30]. Slow oscillations can emerge from non-synaptic interactions, enabling the transitions towards seizure-like states [28,29,31]. Most studies at the network scale take into account the interconnection between neurons through models of synapses [32-38]. In particular, such models can account for the genesis of generalized seizures with spike-and-wave EEG patterns, with mechanisms that critically depend on the balance between GABAergic and glutamate conductances [36,37], as well as cortico-thalamic interactions [36].

An essential question arises at this scale. Network models are constructed from individual models of interconnected neurons. However, assembling these bricks does not guarantee the construction of a biologically relevant network model. One solution is to keep only the emerging behaviors of the network, which display dynamics comparable to those observed experimentally. However, there exist many possibilities of underlying mechanisms to generate the same emerging behavior. One example is the way the seizure appears and spreads. Indeed, the transitions to the ictal state do not occur uniformly in the network. Modeling at this scale makes it possible to observe the collective effects and the propagation of the seizure in the network in many different ways $[12,33,39]$. These observations are based on the bottom-up approach, the network models being constrained by the lower scale (i.e. single neuron). The reverse approach, imposing the phenomenological constraint from the upper scale to build a 
network with a biophysical description, was implemented by Naze and colleagues [40]. Even if the number of biophysical interactions described remains limited, they show how dynamical system theory makes it possible to build a biophysical model on a smaller scale from a framework derived from macroscopic observations.

The construction of networks involves high-dimension models, making it impossible to directly apply the dynamical analysis used in low-dimension systems. To overcome this problem, one can consider global measures or reduce the description of the network's activity to a reduced number of variables. Considering the network as is suited to study the activity on larger scales, which is discussed in the next section.

\section{From mean-field to large scale models}

Mean-Field models describe the activity of a large group of neurons using averaged values of their activity, such as the mean membrane potential or, more often, the firing rate of the population. Some studies have derived these descriptors of the activity from single neuron models $[41,42]$. Another method is to consider that a neuron can represent the mean activity of a population of neurons. It can be used in neuralfield (including space representation) or neural mass model to describe seizures $[33,43,44]$. One of the historical models at this scale has been developed by Wilson and Cowan [45] and has been used to study focal epilepsy through bifurcation analysis $[44,46]$. Keeping the idea of a transfer function that can be used to describe a population activity, a model shows the role of GABAergic dendritic inhibition in the appearance of seizures [47]. Recent work using this model enabled the development of seizure prediction tools based on an active approach: the system's response to a stimulus establishes its proximity to the ictal state [48]. 
These approaches allow us to understand phenomena observable at a global activity scale $[44,49]$. The reduction of networks to mean-field models necessarily leads to the loss of information on certain internal dynamics and collective effects to describe only certain global aspects. But this may be sufficient to characterize seizures. Reducing the number of variables enables a bifurcation analysis. This reduction enables the comparison with phenomenological models.

At such scale of analysis, results are relevant to recordings obtained in humans, using scalp or intracerebral EEGs. In particular, it is possible to study interactions between regions, like thalomo-cortical interactions [50]. It also enables studying particular properties of networks in a phenomenological way [51,52]. At this scale, the temporal dissection exists, and the variation of a parameter or slow variables enables the transitions to the ictal state and its return to a "healthy" regime $[47,50]$. As such models are at the scale of a large region, it is possible to connect them to create a large-scale network describing the whole brain $[53,54]$.

A whole-brain approach using the phenomenological model described previously has been developed [55]. At this level, such models can have important clinical applications. One example is predicting the size of the epileptogenic zone in pharmaco-resistant epilepsies [56-60]. From such a study, it is also possible to highlight network effects in epilepsy $[59,61]$, opening the way to further studies on the dynamics of networks and the possible pathological oscillations that can emerge specifically due to network topology, leading to the ictal state. Another possible outcome of such a modeling approach is the control of seizures through stimulation $[62,63]$. These computational neuroscience methods have developed very rapidly in recent years; they hold great promises regarding translation to the clinic. 


\section{Discussion}

Computational models applied to epilepsy already have a long history [6]. Recent progress in seizure modeling has brought a new understanding of this complex phenomenon. $[8,10,23][8,10,23][8,10,23]$ lt is possible to create direct correspondences with experimentally measurable physical quantities at the level of biophysical interactions, such as variations in ionic concentrations $[8,10,23]$. It is possible to make predictions and design intervention strategies targeting known biophysical pathways. In terms of collective network dynamics, computational modeling shows how the complexity leads to the emergence of a rich repertoire of behaviors, including seizures [44]. At this mesoscopic scale, reduced models have been proposed, describing the relation between electrophysiological activities and recorded signals such as EEG [64]. Networks of mesoscopic models permit the study of the seizure propagations, building the bridge with macroscopic scales and clinical observations.

Thus, the phenomena associated with seizures can be described at different scales. A global understanding of this phenomenon requires this pluralist approach in terms of scale. The complexity of the brain does not allow us to establish a simple relationship between the different scales. However, it seems that fundamental invariant properties are needed, such as at least two distinct time scales: one representing the rapid electrophysiological activity or the variations of measured signals, and a second, slower, at the origin of the transitions between the ictal and non-ictal states, which can be linked to a very wide variety of phenomena depending on the scales. This temporal dissection is included in the phenomenological model, which also opens the way for classification according to the characteristics of the onset and the offset of the seizures through the different types of bifurcations found in the biophysical models at different scales. This classification is consistent with clinical observations where the onset is an 
important criterion [65]. Such an approach offers a direct link between theoretical, computational studies and clinical practice through new tools that have been born in recent years, such as the virtual epileptic patient [7]. It tends to contribute to developing seizure forecasting methods and presurgical evaluation [66].

\section{Acknowledgment}

This project/research has received funding from the European Union's Horizon 2020 Framework Programme for Research and Innovation under the Specific Grant Agreement No. 945539 (Human Brain Project SGA1, SGA2, SGA3) and the Centre National de la Recherche Scientifique (CNRS, France).

\section{References}

[1] Fisher RS, Acevedo C, Arzimanoglou A, Bogacz A, Cross JH, Elger CE, et al. ILAE Official Report: A practical clinical definition of epilepsy. Epilepsia 2014;55:475-82. https://doi.org/10.1111/epi.12550.

[2] Saggio ML, Crisp D, Scott JM, Karoly P, Kuhlmann L, Nakatani M, et al. A taxonomy of seizure dynamotypes. Elife 2020;9. https://doi.org/10.7554/eLife.55632.

[3] Jirsa, Stacey, Quilichini PP, Ivanov AI, Bernard C. On the nature of seizure dynamics. Brain 2014;137:2210-30. https://doi.org/10.1093/brain/awu133.

[4] Saggio ML, Spiegler A, Bernard C, Jirsa VK. Fast-Slow Bursters in the Unfolding of a High Codimension Singularity and the Ultra-slow Transitions of Classes. J Math Neurosci 2017;7:7. https://doi.org/10.1186/s13408-017-00508.

[5] Stefanescu RA, Shivakeshavan RG, Talathi SS. Computational models of 
epilepsy. Seizure 2012;21:748-59.

https://doi.org/10.1016/j.seizure.2012.08.012.

[6] Soltesz I, Staley K. Computational neuroscience in epilepsy. Academic; 2008.

[7] Jirsa VK, Proix T, Perdikis D, Woodman MM, Wang H, Gonzalez-Martinez J, et al. The Virtual Epileptic Patient: Individualized whole-brain models of epilepsy spread. Neuroimage 2017;145:377-88.

https://doi.org/10.1016/J.NEUROIMAGE.2016.04.049.

[8] Depannemaecker D, Ivanov A, Lillo D, Spek L, Bernard C, Jirsa V. A unified physiological framework of transitions between seizures, status epilepticus and depolarization block at the single neuron level. BioRxiv 2020:2020.10.23.352021. https://doi.org/10.1101/2020.10.23.352021.

[9] Toglia P, Ullah G. Mitochondrial dysfunction and role in spreading depolarization and seizure. J Comput Neurosci 2019;47:91-108. https://doi.org/10.1007/s10827-019-00724-6.

[10] Wei Y, Ullah G, Schiff SJ. Unification of neuronal spikes, seizures, and spreading depression. J Neurosci 2014;34:11733-43.

[11] Wei Y, Ullah G, Ingram J, Schiff SJ. Oxygen and seizure dynamics: II. Computational modeling. J Neurophysiol 2014;112:213-23. https://doi.org/10.1152/jn.00541.2013.

[12] Depannemaecker D, Carlu M, Destexhe A. Response dynamics of spiking network models to incoming seizure-like perturbation. Submitted 2021.

[13] Ritter P, Schirner M, Mcintosh AR, Jirsa VK. The Virtual Brain Integrates Computational Modeling and Multimodal Neuroimaging. Brain Connect 
2013;3:121-45. https://doi.org/10.1089/brain.2012.0120.

[14] Sip V, Guye M, Bartolomei F, Jirsa V. Computational modeling of seizure spread on a cortical surface. BioRxiv 2020:2020.12.22.423957. https://doi.org/10.1101/2020.12.22.423957.

[15] Cosandier-Rimélé D, Merlet I, Bartolomei F, Badier JM, Wendling F. Computational modeling of epileptic activity: From cortical sources to EEG signals. J Clin Neurophysiol 2010;27:465-70. https://doi.org/10.1097/WNP.0b013e3182005dcd.

[16] Bertram R, Rubin JE. Multi-timescale systems and fast-slow analysis. Math Biosci 2017;287:105-21. https://doi.org/10.1016/j.mbs.2016.07.003.

[17] Izhikevich E. Dynamical Systems In Neuroscience : The Geometry of Excitability and Bursting. MIT Press 2007.

[18] El Houssaini K, Ivanov Al, Bernard C, Jirsa VK. Seizures, refractory status epilepticus, and depolarization block as endogenous brain activities. Phys Rev E 2015;91:010701. https://doi.org/10.1103/PhysRevE.91.010701.

[19] Houssaini K El, Bernard C, Jirsa VK. The epileptor model: A systematic mathematical analysis linked to the dynamics of seizures, refractory status epilepticus, and depolarization block. ENeuro 2020;7. https://doi.org/10.1523/ENEURO.0485-18.2019.

[20] Santhakumar V, Aradi I, Soltesz I. Role of mossy fiber sprouting and mossy cell loss in hyperexcitability: A network model of the dentate gyrus incorporating cell types and axonal topography. J Neurophysiol 2005;93:437-53. https://doi.org/10.1152/jn.00777.2004. 
[21] Tejada J, Garcia-Cairasco N, Roque AC. Combined Role of Seizure-Induced Dendritic Morphology Alterations and Spine Loss in Newborn Granule Cells with Mossy Fiber Sprouting on the Hyperexcitability of a Computer Model of the Dentate Gyrus. PLoS Comput Biol 2014;10:1003601. https://doi.org/10.1371/journal.pcbi.1003601.

[22] Izhikevich EM. Which Model to Use for Cortical Spiking Neurons? IEEE Trans NEURAL NETWORKS 2004;15:1063.

https://doi.org/10.1109/TNN.2004.832719.

[23] Cressman JR, Ullah G, Ziburkus J, Schiff SJ, Barreto E. The influence of sodium and potassium dynamics on excitability, seizures, and the stability of persistent states: I. Single neuron dynamics. J Comput Neurosci 2009;26:15970. https://doi.org/10.1007/s10827-008-0132-4.

[24] Hodgkin, A. L., Huxley F. A quantitative description of membrane current and its application to conduction and excitation in nerve. J Physiol 1952;117:50044.

[25] Ullah G, Schiff SJ. Assimilating seizure dynamics. PLoS Comput Biol 2010;6:e1000776. https://doi.org/10.1371/journal.pcbi.1000776.

[26] Ingram J, Zhang C, Cressman JR, Hazra A, Wei Y, Koo YE, et al. Oxygen and seizure dynamics: I. experiments. J Neurophysiol 2014;112:205-12. https://doi.org/10.1152/jn.00540.2013.

[27] Chizhov A V., Zefirov A V., Amakhin D V., Smirnova EY, Zaitsev A V. Minimal model of interictal and ictal discharges "Epileptor-2." PLOS Comput Biol 2018;14:e1006186. https://doi.org/10.1371/journal.pcbi.1006186. 
[28] de Almeida A-CG, Rodrigues AM, Scorza FA, Cavalheiro EA, Teixeira HZ, Duarte MA, et al. Mechanistic hypotheses for nonsynaptic epileptiform activity induction and its transition from the interictal to ictal state-Computational simulation. Epilepsia 2008;49:1908-24. https://doi.org/10.1111/j.15281167.2008.01686.x.

[29] Rodrigues AM, Santos LEC, Covolan L, Hamani C, Almeida A-CG. pH during non-synaptic epileptiform activity—computational simulations. Phys Biol 2015;12:056007. https://doi.org/10.1088/1478-3975/12/5/056007.

[30] Sheheitli $\mathrm{H}$, Jirsa VK. A mathematical model of ephaptic interactions in neuronal fiber pathways: Could there be more than transmission along the tracts? Netw Neurosci 2020;4:595-610. https://doi.org/10.1162/netn_a_00134.

[31] Bikson M, Ruiz-Nuño A, Miranda D, Kronberg G, Jiruska P, Fox JE, et al. Synaptic transmission modulates while non-synaptic processes govern the transition from pre-ictal to seizure activity in vitro. BioRxiv 2018:280321. https://doi.org/10.1101/280321.

[32] Naze S, Bernard C, Jirsa V. Computational Modeling of Seizure Dynamics Using Coupled Neuronal Networks: Factors Shaping Epileptiform Activity. PLOS Comput Biol 2015;11:e1004209.

https://doi.org/10.1371/journal.pcbi.1004209.

[33] Liou JY, Smith EH, Bateman LM, Bruce SL, McKhann GM, Goodman RR, et al. A model for focal seizure onset, propagation, evolution, and progression. Elife 2020;9. https://doi.org/10.7554/eLife.50927.

[34] Kim CM, Nykamp DQ. The influence of depolarization block on seizure-like activity in networks of excitatory and inhibitory neurons. J Comput Neurosci 
2017;43:65-79. https://doi.org/10.1007/s10827-017-0647-7.

[35] Jacob T, Lillis KP, Wang Z, Swiercz W, Rahmati N, Staley KJ. A proposed mechanism for spontaneous transitions between interictal and ictal activity. $\mathrm{J}$ Neurosci 2019;39:557-75. https://doi.org/10.1523/JNEUROSCI.0719-17.2018.

[36] Destexhe A. Spike-and-wave oscillations based on the properties of GABA(B) receptors. J Neurosci 1998;18:9099-111. https://doi.org/10.1523/jneurosci.1821-09099.1998.

[37] Destexhe A. Can GABA A conductances explain the fast oscillation frequency of absence seizures in rodents? Eur J Neurosci 1999;11:2175-81. https://doi.org/10.1046/j.1460-9568.1999.00660.x.

[38] Destexhe A. Corticothalamic Feedback: A Key to Explain Absence Seizures. Comput. Neurosci. Epilepsy, Elsevier Inc.; 2008, p. 184-214. https://doi.org/10.1016/B978-012373649-9.50016-8.

[39] Y. Ho EC, Truccolo W. Interaction between synaptic inhibition and glialpotassium dynamics leads to diverse seizure transition modes in biophysical models of human focal seizures. J Comput Neurosci 2016;41:225-44. https://doi.org/10.1007/s10827-016-0615-7.

[40] Naze S, Bernard C, Jirsa V. Computational Modeling of Seizure Dynamics Using Coupled Neuronal Networks: Factors Shaping Epileptiform Activity. PLOS Comput Biol 2015;11:e1004209.

https://doi.org/10.1371/journal.pcbi.1004209.

[41] Carlu M, Chehab O, Dalla Porta XL, Depannemaecker D, Héricé XC, Jedynak $\mathrm{M}$, et al. A mean-field approach to the dynamics of networks of complex 
neurons, from nonlinear Integrate-and-Fire to Hodgkin-Huxley models. J Neurophysiol 2020;123:1042-51. https://doi.org/10.1152/JN.00399.2019.

[42] Montbrió E, Pazó D, Roxin A. Macroscopic Description for Networks of Spiking Neurons. Phys Rev X 2015;5:021028.

https://doi.org/10.1103/PhysRevX.5.021028.

[43] Buchin A, Kerr CC, Huberfeld G, Miles R, Gutkin B. Adaptation and inhibition control pathological synchronization in a model of focal epileptic seizure. ENeuro 2018;5:19-37. https://doi.org/10.1523/ENEURO.0019-18.2018.

[44] Kim CM, Nykamp DQ. The influence of depolarization block on seizure-like activity in networks of excitatory and inhibitory neurons. J Comput Neurosci 2017;43:65-79. https://doi.org/10.1007/s10827-017-0647-7.

[45] Wilson HR, Cowan JD. Excitatory and Inhibitory Interactions in Localized Populations of Model Neurons. Biophys J 1972;12:1-24. https://doi.org/10.1016/S0006-3495(72)86068-5.

[46] Meijer HGE, Eissa TL, Kiewiet B, Neuman JF, Schevon CA, Emerson RG, et al. Modeling focal epileptic activity in the Wilson-cowan model with depolarization block. J Math Neurosci 2015;5:7. https://doi.org/10.1186/s13408-015-0019-4.

[47] Wendling F, Bartolomei F, Bellanger JJ, Chauvel P. Epileptic fast activity can be explained by a model of impaired GABAergic dendritic inhibition. Eur $\mathrm{J}$ Neurosci 2002;15:1499-508. https://doi.org/10.1046/j.14609568.2002.01985.x.

[48] Carvalho VR, Moraes MFD, Cash SS, Mendes EMAM. Active probing to 
highlight approaching transitions to ictal states in coupled neural mass models. PLoS Comput Biol 2021;17:e1008377.

https://doi.org/10.1371/journal.pcbi.1008377.

[49] Takeshita D, Sato YD, Bahar S. Transitions between multistable states as a model of epileptic seizure dynamics. Phys Rev E - Stat Nonlinear, Soft Matter Phys 2007;75. https://doi.org/10.1103/PhysRevE.75.051925.

[50] Marten F, Rodrigues S, Suffczynski P, Richardson MP, Terry JR. Derivation and analysis of an ordinary differential equation mean-field model for studying clinically recorded epilepsy dynamics. Phys Rev E - Stat Nonlinear, Soft Matter Phys 2009;79:021911. https://doi.org/10.1103/PhysRevE.79.021911.

[51] Benjamin O, Fitzgerald THB, Ashwin P, Tsaneva-Atanasova K, Chowdhury F, Richardson MP, et al. A phenomenological model of seizure initiation suggests network structure may explain seizure frequency in idiopathic generalised epilepsy. J Math Neurosci 2012;2:1. https://doi.org/10.1186/2190-8567-2-1.

[52] Terry JR, Benjamin O, Richardson MP. Seizure generation: The role of nodes and networks. Epilepsia 2012;53:e166-9. https://doi.org/10.1111/j.15281167.2012.03560.x.

[53] Melozzi F, Woodman MM, Jirsa VK, Bernard C. The virtual mouse brain: A computational neuroinformatics platform to study whole mouse brain dynamics. ENeuro 2017;4. https://doi.org/10.1523/ENEURO.0111-17.2017.

[54] Goldman J, Kusch L, Hazalyalcinkaya B, Depannemaecker D, Nghiem T-A, Jirsa V, et al. Brain-scale emergence of slow-wave synchrony and highly responsive asynchronous states based on biologically realistic population models simulated in The Virtual Brain. BioRxiv 2020:2020.12.28.424574. 
https://doi.org/10.1101/2020.12.28.424574.

[55] Olmi S, Petkoski S, Guye M, Bartolomei F, Jirsa V. Controlling seizure propagation in large-scale brain networks. PLOS Comput Biol 2019;15:e1006805. https://doi.org/10.1371/journal.pcbi.1006805.

[56] Junges L, Lopes MA, Terry JR, Goodfellow M. The role that choice of model plays in predictions for epilepsy surgery. Sci Rep 2019;9. https://doi.org/10.1038/s41598-019-43871-7.

[57] Kini LG, Bernabei JM, Mikhail F, Hadar P, Shah P, Khambhati AN, et al. Virtual resection predicts surgical outcome for drug-resistant epilepsy. Brain 2019;142:3892-905. https://doi.org/10.1093/brain/awz303.

[58] An S, Bartolomei F, Guye M, Jirsa V. Optimization of surgical intervention outside the epileptogenic zone in the virtual epileptic patient (VEP). PLoS Comput Biol 2019;15. https://doi.org/10.1371/journal.pcbi.1007051.

[59] Jirsa VK, Proix T, Perdikis D, Woodman MM, Wang H, Bernard C, et al. The Virtual Epileptic Patient: Individualized whole-brain models of epilepsy spread. Neuroimage 2017;145:377-88.

https://doi.org/10.1016/j.neuroimage.2016.04.049.

[60] Lopes MA, Richardson MP, Abela E, Rummel C, Schindler K, Goodfellow M, et al. An optimal strategy for epilepsy surgery: Disruption of the rich-club? PLoS Comput Biol 2017;13:e1005637. https://doi.org/10.1371/journal.pcbi.1005637.

[61] Proix T, Bartolomei F, Chauvel P, Bernard C, Jirsa VK. Permittivity Coupling across Brain Regions Determines Seizure Recruitment in Partial Epilepsy. J Neurosci 2014;34:15009-21. https://doi.org/10.1523/JNEUROSCI.1570- 
14.2014.

[62] Ching S, Brown EN, Kramer MA. Distributed control in a mean-field cortical network model: Implications for seizure suppression. Phys Rev E - Stat Nonlinear, Soft Matter Phys 2012;86.

https://doi.org/10.1103/PhysRevE.86.021920.

[63] Ashourvan A, Pequito S, Khambhati AN, Mikhail F, Baldassano SN, Davis KA, et al. Model-based design for seizure control by stimulation. J Neural Eng 2020;17. https://doi.org/10.1088/1741-2552/ab7a4e.

[64] Blenkinsop A, Valentin A, Richardson MP, Terry JR. The dynamic evolution of focal-onset epilepsies - combining theoretical and clinical observations. Eur J Neurosci 2012;36:2188-200. https://doi.org/10.1111/j.14609568.2012.08082.x.

[65] Lagarde S, Buzori S, Trebuchon A, Carron R, Scavarda D, Milh M, et al. The repertoire of seizure onset patterns in human focal epilepsies: Determinants and prognostic values. Epilepsia 2019;60:85-95. https://doi.org/10.1111/epi.14604.

[66] Kuhlmann L, Grayden DB, Wendling F, Schiff SJ. Role of multiple-scale modeling of epilepsy in seizure forecasting. J Clin Neurophysiol 2015;32:2206. https://doi.org/10.1097/WNP.0000000000000149.

[2] Saggio ML, Crisp D, Scott JM, Karoly P, Kuhlmann L, Nakatani M, et al. A taxonomy of seizure dynamotypes. Elife 2020;9. https://doi.org/10.7554/eLife.55632. 
[3] Jirsa, Stacey, Quilichini PP, Ivanov AI, Bernard C. On the nature of seizure dynamics. Brain 2014;137:2210-30. https://doi.org/10.1093/brain/awu133.

[4] Saggio ML, Spiegler A, Bernard C, Jirsa VK. Fast-Slow Bursters in the Unfolding of a High Codimension Singularity and the Ultra-slow Transitions of Classes. J Math Neurosci 2017;7:7. https://doi.org/10.1186/s13408-017-00508.

[5] Stefanescu RA, Shivakeshavan RG, Talathi SS. Computational models of epilepsy. Seizure 2012;21:748-59. https://doi.org/10.1016/j.seizure.2012.08.012.

[6] Soltesz I, Staley K. Computational neuroscience in epilepsy. Academic; 2008.

[7] Jirsa VK, Proix T, Perdikis D, Woodman MM, Wang H, Gonzalez-Martinez J, et al. The Virtual Epileptic Patient: Individualized whole-brain models of epilepsy spread. Neuroimage 2017;145:377-88. https://doi.org/10.1016/J.NEUROIMAGE.2016.04.049.

[8] Depannemaecker D, Ivanov A, Lillo D, Spek L, Bernard C, Jirsa V. A unified physiological framework of transitions between seizures, status epilepticus and depolarization block at the single neuron level. BioRxiv 2020:2020.10.23.352021. https://doi.org/10.1101/2020.10.23.352021.

[9] Toglia P, Ullah G. Mitochondrial dysfunction and role in spreading depolarization and seizure. J Comput Neurosci 2019;47:91-108. https://doi.org/10.1007/s10827-019-00724-6.

[10] Wei Y, Ullah G, Schiff SJ. Unification of neuronal spikes, seizures, and spreading depression. J Neurosci 2014;34:11733-43. 
[11] Wei Y, Ullah G, Ingram J, Schiff SJ. Oxygen and seizure dynamics: II. Computational modeling. J Neurophysiol 2014;112:213-23. https://doi.org/10.1152/jn.00541.2013.

[12] Depannemaecker D, Carlu M, Destexhe A. Response dynamics of spiking network models to incoming seizure-like perturbation. Submitted 2021.

[13] Ritter P, Schirner M, Mcintosh AR, Jirsa VK. The Virtual Brain Integrates Computational Modeling and Multimodal Neuroimaging. Brain Connect 2013;3:121-45. https://doi.org/10.1089/brain.2012.0120.

[14] Sip V, Guye M, Bartolomei F, Jirsa V. Computational modeling of seizure spread on a cortical surface. BioRxiv 2020:2020.12.22.423957. https://doi.org/10.1101/2020.12.22.423957.

[15] Cosandier-Rimélé D, Merlet I, Bartolomei F, Badier JM, Wendling F. Computational modeling of epileptic activity: From cortical sources to EEG signals. J Clin Neurophysiol 2010;27:465-70. https://doi.org/10.1097/WNP.0b013e3182005dcd.

[16] Bertram R, Rubin JE. Multi-timescale systems and fast-slow analysis. Math Biosci 2017;287:105-21. https://doi.org/10.1016/j.mbs.2016.07.003.

[17] Izhikevich E. Dynamical Systems In Neuroscience: The Geometry of Excitability and Bursting. MIT Press 2007.

[18] El Houssaini K, Ivanov AI, Bernard C, Jirsa VK. Seizures, refractory status epilepticus, and depolarization block as endogenous brain activities. Phys Rev E 2015;91:010701. https://doi.org/10.1103/PhysRevE.91.010701.

[19] Houssaini K El, Bernard C, Jirsa VK. The epileptor model: A systematic 
mathematical analysis linked to the dynamics of seizures, refractory status epilepticus, and depolarization block. ENeuro 2020;7. https://doi.org/10.1523/ENEURO.0485-18.2019.

[20] Santhakumar V, Aradi I, Soltesz I. Role of mossy fiber sprouting and mossy cell loss in hyperexcitability: A network model of the dentate gyrus incorporating cell types and axonal topography. J Neurophysiol 2005;93:437-53. https://doi.org/10.1152/jn.00777.2004.

[21] Tejada J, Garcia-Cairasco N, Roque AC. Combined Role of Seizure-Induced Dendritic Morphology Alterations and Spine Loss in Newborn Granule Cells with Mossy Fiber Sprouting on the Hyperexcitability of a Computer Model of the Dentate Gyrus. PLoS Comput Biol 2014;10:1003601. https://doi.org/10.1371/journal.pcbi.1003601.

[22] Izhikevich EM. Which Model to Use for Cortical Spiking Neurons? IEEE Trans NEURAL NETWORKS 2004;15:1063.

https://doi.org/10.1109/TNN.2004.832719.

[23] Cressman JR, Ullah G, Ziburkus J, Schiff SJ, Barreto E. The influence of sodium and potassium dynamics on excitability, seizures, and the stability of persistent states: I. Single neuron dynamics. J Comput Neurosci 2009;26:159_ 70. https://doi.org/10.1007/s10827-008-0132-4.

[24] Hodgkin, A. L., Huxley F. A quantitative description of membrane current and its application to conduction and excitation in nerve. J Physiol 1952;117:50044.

[25] Ullah G, Schiff SJ. Assimilating seizure dynamics. PLoS Comput Biol 2010;6:e1000776. https://doi.org/10.1371/journal.pcbi.1000776. 
[26] Ingram J, Zhang C, Cressman JR, Hazra A, Wei Y, Koo YE, et al. Oxygen and seizure dynamics: I. experiments. J Neurophysiol 2014;112:205-12. https://doi.org/10.1152/jn.00540.2013.

[27] Chizhov A V., Zefirov A V., Amakhin D V., Smirnova EY, Zaitsev A V. Minimal model of interictal and ictal discharges "Epileptor-2." PLOS Comput Biol 2018;14:e1006186. https://doi.org/10.1371/journal.pcbi.1006186.

[28] de Almeida A-CG, Rodrigues AM, Scorza FA, Cavalheiro EA, Teixeira HZ, Duarte MA, et al. Mechanistic hypotheses for nonsynaptic epileptiform activity induction and its transition from the interictal to ictal state-Computational simulation. Epilepsia 2008;49:1908-24. https://doi.org/10.1111/j.15281167.2008.01686.x.

[29] Rodrigues AM, Santos LEC, Covolan L, Hamani C, Almeida A-CG. pH during non-synaptic epileptiform activity—computational simulations. Phys Biol 2015;12:056007. https://doi.org/10.1088/1478-3975/12/5/056007.

[30] Sheheitli H, Jirsa VK. A mathematical model of ephaptic interactions in neuronal fiber pathways: Could there be more than transmission along the tracts? Netw Neurosci 2020;4:595-610. https://doi.org/10.1162/netn_a_00134.

[31] Bikson M, Ruiz-Nuño A, Miranda D, Kronberg G, Jiruska P, Fox JE, et al. Synaptic transmission modulates while non-synaptic processes govern the transition from pre-ictal to seizure activity in vitro. BioRxiv 2018:280321. https://doi.org/10.1101/280321.

[32] Naze S, Bernard C, Jirsa V. Computational Modeling of Seizure Dynamics Using Coupled Neuronal Networks: Factors Shaping Epileptiform Activity. PLOS Comput Biol 2015;11:e1004209. 
https://doi.org/10.1371/journal.pcbi.1004209.

[33] Liou JY, Smith EH, Bateman LM, Bruce SL, McKhann GM, Goodman RR, et al. A model for focal seizure onset, propagation, evolution, and progression. Elife 2020;9. https://doi.org/10.7554/eLife.50927.

[34] Kim CM, Nykamp DQ. The influence of depolarization block on seizure-like activity in networks of excitatory and inhibitory neurons. J Comput Neurosci 2017;43:65-79. https://doi.org/10.1007/s10827-017-0647-7.

[35] Jacob T, Lillis KP, Wang Z, Swiercz W, Rahmati N, Staley KJ. A proposed mechanism for spontaneous transitions between interictal and ictal activity. $\mathrm{J}$ Neurosci 2019;39:557-75. https://doi.org/10.1523/JNEUROSCI.0719-17.2018.

[36] Destexhe A. Spike-and-wave oscillations based on the properties of GABA(B) receptors. J Neurosci 1998;18:9099-111. https://doi.org/10.1523/jneurosci.1821-09099.1998.

[37] Destexhe A. Can GABA A conductances explain the fast oscillation frequency of absence seizures in rodents? Eur J Neurosci 1999;11:2175-81. https://doi.org/10.1046/j.1460-9568.1999.00660.x.

[38] Destexhe A. Corticothalamic Feedback: A Key to Explain Absence Seizures. Comput. Neurosci. Epilepsy, Elsevier Inc.; 2008, p. 184-214. https://doi.org/10.1016/B978-012373649-9.50016-8.

[39] Y. Ho EC, Truccolo W. Interaction between synaptic inhibition and glialpotassium dynamics leads to diverse seizure transition modes in biophysical models of human focal seizures. J Comput Neurosci 2016;41:225-44. https://doi.org/10.1007/s10827-016-0615-7. 
[40] Naze S, Bernard C, Jirsa V. Computational Modeling of Seizure Dynamics Using Coupled Neuronal Networks: Factors Shaping Epileptiform Activity. PLOS Comput Biol 2015;11:e1004209.

https://doi.org/10.1371/journal.pcbi.1004209.

[41] Carlu M, Chehab O, Dalla Porta XL, Depannemaecker D, Héricé XC, Jedynak $\mathrm{M}$, et al. A mean-field approach to the dynamics of networks of complex neurons, from nonlinear Integrate-and-Fire to Hodgkin-Huxley models. J Neurophysiol 2020;123:1042-51. https://doi.org/10.1152/JN.00399.2019.

[42] Montbrió E, Pazó D, Roxin A. Macroscopic Description for Networks of Spiking Neurons. Phys Rev X 2015;5:021028.

https://doi.org/10.1103/PhysRevX.5.021028.

[43] Buchin A, Kerr CC, Huberfeld G, Miles R, Gutkin B. Adaptation and inhibition control pathological synchronization in a model of focal epileptic seizure. ENeuro 2018;5:19-37. https://doi.org/10.1523/ENEURO.0019-18.2018.

[44] Kim CM, Nykamp DQ. The influence of depolarization block on seizure-like activity in networks of excitatory and inhibitory neurons. J Comput Neurosci 2017;43:65-79. https://doi.org/10.1007/s10827-017-0647-7.

[45] Wilson HR, Cowan JD. Excitatory and Inhibitory Interactions in Localized Populations of Model Neurons. Biophys J 1972;12:1-24. https://doi.org/10.1016/S0006-3495(72)86068-5.

[46] Meijer HGE, Eissa TL, Kiewiet B, Neuman JF, Schevon CA, Emerson RG, et al. Modeling focal epileptic activity in the Wilson-cowan model with depolarization block. J Math Neurosci 2015;5:7. https://doi.org/10.1186/s13408-015-0019-4. 
[47] Wendling F, Bartolomei F, Bellanger JJ, Chauvel P. Epileptic fast activity can be explained by a model of impaired GABAergic dendritic inhibition. Eur $\mathrm{J}$ Neurosci 2002;15:1499-508. https://doi.org/10.1046/j.14609568.2002.01985.x.

[48] Carvalho VR, Moraes MFD, Cash SS, Mendes EMAM. Active probing to highlight approaching transitions to ictal states in coupled neural mass models. PLoS Comput Biol 2021;17:e1008377. https://doi.org/10.1371/journal.pcbi.1008377.

[49] Takeshita D, Sato YD, Bahar S. Transitions between multistable states as a model of epileptic seizure dynamics. Phys Rev E - Stat Nonlinear, Soft Matter Phys 2007;75. https://doi.org/10.1103/PhysRevE.75.051925.

[50] Marten F, Rodrigues S, Suffczynski P, Richardson MP, Terry JR. Derivation and analysis of an ordinary differential equation mean-field model for studying clinically recorded epilepsy dynamics. Phys Rev E - Stat Nonlinear, Soft Matter Phys 2009;79:021911. https://doi.org/10.1103/PhysRevE.79.021911.

[51] Benjamin O, Fitzgerald THB, Ashwin P, Tsaneva-Atanasova K, Chowdhury F, Richardson MP, et al. A phenomenological model of seizure initiation suggests network structure may explain seizure frequency in idiopathic generalised epilepsy. J Math Neurosci 2012;2:1. https://doi.org/10.1186/2190-8567-2-1.

[52] Terry JR, Benjamin O, Richardson MP. Seizure generation: The role of nodes and networks. Epilepsia 2012;53:e166-9. https://doi.org/10.1111/j.15281167.2012.03560.x.

[53] Melozzi F, Woodman MM, Jirsa VK, Bernard C. The virtual mouse brain: A computational neuroinformatics platform to study whole mouse brain dynamics. 
ENeuro 2017;4. https://doi.org/10.1523/ENEURO.0111-17.2017.

[54] Goldman J, Kusch L, Hazalyalcinkaya B, Depannemaecker D, Nghiem T-A, Jirsa $\mathrm{V}$, et al. Brain-scale emergence of slow-wave synchrony and highly responsive asynchronous states based on biologically realistic population models simulated in The Virtual Brain. BioRxiv 2020:2020.12.28.424574. https://doi.org/10.1101/2020.12.28.424574.

[55] Olmi S, Petkoski S, Guye M, Bartolomei F, Jirsa V. Controlling seizure propagation in large-scale brain networks. PLOS Comput Biol 2019;15:e1006805. https://doi.org/10.1371/journal.pcbi.1006805.

[56] Junges L, Lopes MA, Terry JR, Goodfellow M. The role that choice of model plays in predictions for epilepsy surgery. Sci Rep 2019;9. https://doi.org/10.1038/s41598-019-43871-7.

[57] Kini LG, Bernabei JM, Mikhail F, Hadar P, Shah P, Khambhati AN, et al. Virtual resection predicts surgical outcome for drug-resistant epilepsy. Brain 2019;142:3892-905. https://doi.org/10.1093/brain/awz303.

[58] An S, Bartolomei F, Guye M, Jirsa V. Optimization of surgical intervention outside the epileptogenic zone in the virtual epileptic patient (VEP). PLoS Comput Biol 2019;15. https://doi.org/10.1371/journal.pcbi.1007051.

[59] Jirsa VK, Proix T, Perdikis D, Woodman MM, Wang H, Bernard C, et al. The Virtual Epileptic Patient: Individualized whole-brain models of epilepsy spread. Neuroimage 2017;145:377-88.

https://doi.org/10.1016/j.neuroimage.2016.04.049.

[60] Lopes MA, Richardson MP, Abela E, Rummel C, Schindler K, Goodfellow M, et 
al. An optimal strategy for epilepsy surgery: Disruption of the rich-club? PLoS Comput Biol 2017;13:e1005637. https://doi.org/10.1371/journal.pcbi.1005637.

[61] Proix T, Bartolomei F, Chauvel P, Bernard C, Jirsa VK. Permittivity Coupling across Brain Regions Determines Seizure Recruitment in Partial Epilepsy. J Neurosci 2014;34:15009-21. https://doi.org/10.1523/JNEUROSCI.157014.2014.

[62] Ching S, Brown EN, Kramer MA. Distributed control in a mean-field cortical network model: Implications for seizure suppression. Phys Rev E - Stat Nonlinear, Soft Matter Phys 2012;86.

https://doi.org/10.1103/PhysRevE.86.021920.

[63] Ashourvan A, Pequito S, Khambhati AN, Mikhail F, Baldassano SN, Davis KA, et al. Model-based design for seizure control by stimulation. J Neural Eng 2020;17. https://doi.org/10.1088/1741-2552/ab7a4e.

[64] Blenkinsop A, Valentin A, Richardson MP, Terry JR. The dynamic evolution of focal-onset epilepsies - combining theoretical and clinical observations. Eur $\mathrm{J}$ Neurosci 2012;36:2188-200. https://doi.org/10.1111/j.14609568.2012.08082.x.

[65] Lagarde S, Buzori S, Trebuchon A, Carron R, Scavarda D, Milh M, et al. The repertoire of seizure onset patterns in human focal epilepsies: Determinants and prognostic values. Epilepsia 2019;60:85-95. https://doi.org/10.1111/epi.14604.

[66] Kuhlmann L, Grayden DB, Wendling F, Schiff SJ. Role of multiple-scale modeling of epilepsy in seizure forecasting. J Clin Neurophysiol 2015;32:2206. https://doi.org/10.1097/WNP.0000000000000149. 